\title{
A New Alternative Technique for Preperitoneal Inguinal Hernia Repair: Using Groin Innominate Fascial Island Flap
}

\author{
Feyzi Kurt $^{1 *}$, Musa Abeş ${ }^{2}$ \\ ${ }^{1}$ Department of General Surgery, Adiyaman University Training and Research Hospital, Adiyaman, Turkey \\ ${ }^{2}$ Department of Pediatric Surgery, Adiyaman University Training and Research Hospital, Adiyaman, Turkey \\ Email: *feyzikurt02@hotmail.com
}

Received March 24, 2012; revised April 19, 2012; accepted June 9, 2012

\begin{abstract}
Aim: Despite the fact that reported low recurrence rates and improvement results, optimal surgical technique for inguinal hernia repair was not found and recurrence was not eliminated. We used firstly groin innominate fascial island flap to reinforce the weak inguinal tissue for the repair. Methods: From the February 2010 to December 2010, 15 patients (13 male and 2 female) with inguinal hernia underwent repair with using island groin innominate fascial flap. The follow-up period ranged from 8 to 12 months (mean, 10 months). Results: Seroma developed in one case. Others had not any complications. Conclusion: Because of our technique avoids from the complications and drawbacks of the prosthetic mesh, autograft and abdominal wall flaps, we think that the groin deep fascial flap can be a good alternative to prosthetic meshes for reconstruction of inguinal hernia repair.
\end{abstract}

Keywords: Inguinal Hernia; Innominate Fascia; Preperitoneal Repair

\section{Introduction}

The management of inguinal hernias is one of the most challenging problems in surgery. Different surgical techniques are proposed for the treatment of inguinal hernias. Despite reported low recurrence rates and improvement results, the optimal surgical technique has not been found and recurrence was not eliminated $[1,2]$. Several prosthetic materials, fascial flaps and aponeurotic flaps have been used for reinforcement of weak inguinal tissues and eliminating recurrence [1-11]. We used an open anterior procedure which combined with groin innominate fascial island flap to reinforce the weak inguinal tissue for the repair. Our aim of this combination is to decrease the recurrence rate of the open anterior procedure and avoid complications of the mesh application.

\section{Patients and Methods}

From the February 2010 to December 2010, 15 patients (13 male and 2 female) with inguinal hernia underwent repair according to our technique. Their ages ranged from 26 - 60 years with a mean of 36.6 years. 12 of the patients had direct inguinal hernias, and 3 had indirect inguinal hernias. All cases received general anesthesia.

"Corresponding author.
Analgesics were given only when necessary; antibiotics were continued for 5 days. Ambulation was allowed on the first postoperative day and normal physical activity was allowed on the tenth postoperative day. Strenuous activity was allowed four weeks after the operation.

\section{Surgical Technique}

Approximately 8 - $10 \mathrm{~cm}$ long oblique inguinal skin incision line and flap dimension were outlined with marking pen. The flap size was ranged from $8 \times 6 \mathrm{~cm}$ to $12 \times 10$ $\mathrm{cm}$ (mean, $8 \times 10 \mathrm{~cm}$ ). Inferior side of the flap was designed $2 \mathrm{~cm}$ below the inguinal ligament and medial side was designed from the midpoint of the inguinal ligament (Figure 1). After the skin incision, superficial circumflex iliac artery and vein were identified easily under low magnification with a loop. Suprafascial blunt dissection was made with scissors to reach flap outline. Innominate fascia at the lateral side of the flap was incised and a fascial flap was elevated lateral to medial direction (Figures 2 and 3). No attempt was made to incisize around the pedicle for not injuring it. The hernias were repaired according to the classic Shouldice technique. After the repair of the floor of the inguinal canal, that flap was transported through the dissected tunnel, sized only for just allowing pedicle pass, beneath the inguinal ligament 

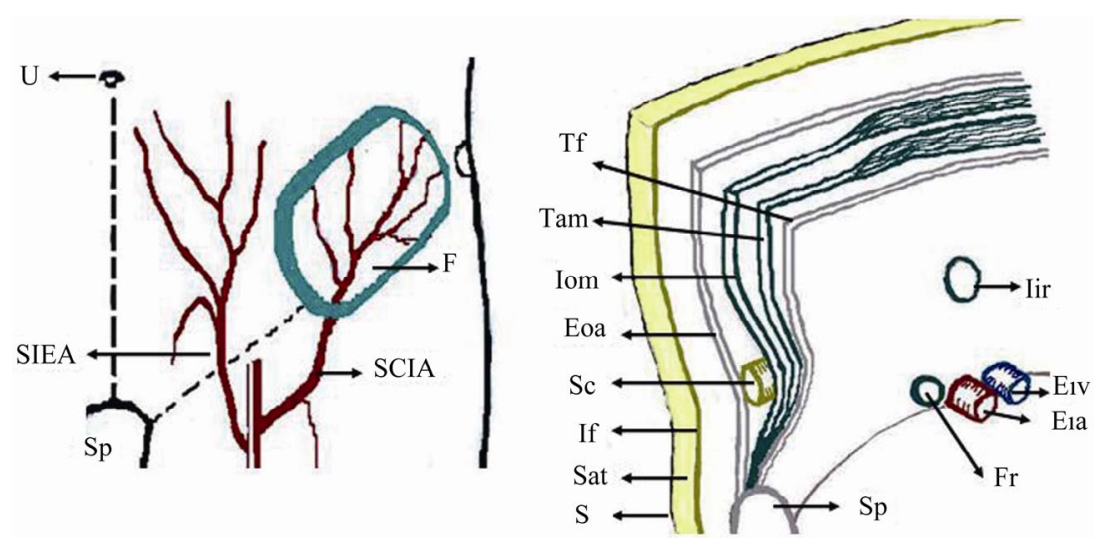

Figure 1. Schematic diagram of inguinal area and components of abdominal wall. Left: Inguinal area and design of the flap. Right: Components of abdominal wall. U: umbilicus; Sp: Symphysis pubis; SIEA: Superficial inferior epigastric artery; SCIA, Superficial circumflex iliac artery; F: Flap; Tf: Tranversalis fascia; Tam: Transversus abdominis muscle; Iom: Internal oblique muscle; Eoa: External oblique aponeurosis; Sc: Spermatic cord; If: Innominate fascia; Sat: Subcutaneous adipose tissue; S: Skin; Iir: Internal inguinal ring; Eia: External iliac artery; Eiv: External iliac vein; Fr: Femoral ring.
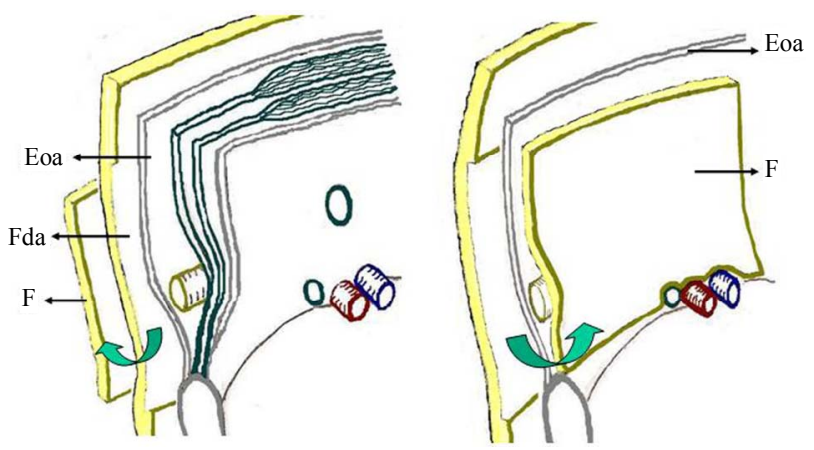

Figure 2. Schematic diagram of elevation and placement of the flap. Left: Elevation of the flap. Right: Placement of the flap covered and sufficiently overlapped all potential hernial sites. Eoa: External oblique aponeurosis; Fda: Flap donor area; F: Flap.

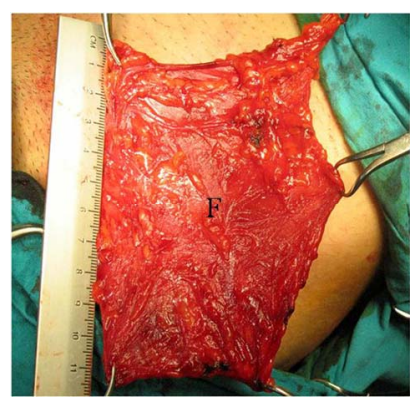

Figure 3. Intraoperative view; innominate fascial flap was elevated at the left inguinal area. F: Flap.

and introduced beneath external oblique aponeurosis. Placement of the flap covered and sufficiently overlapped all potential hernial sites (Figure 3, right and Figure 4). An outline of the flap was sutured superiorly to the internal oblique muscle and the anterior sheet of the rectus abdominus muscle and inferiorly lacunar ligament with $2 / 0$ polyglycolic acid sutures (Figure 4). External

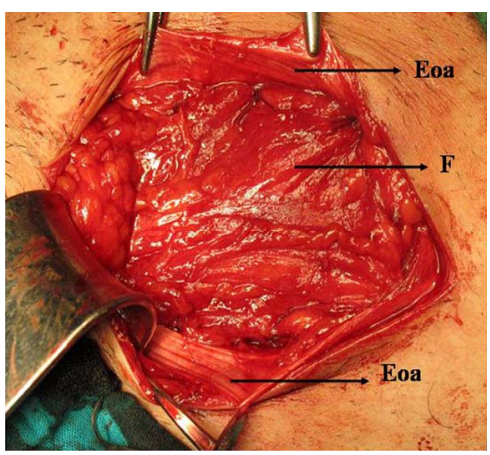

Figure 4. Intraoperative view; placement of the flap on the all potential hernial sites and outline of the flap was sutured superiorly to the internal oblique muscle and the anterior sheet of the rectus abdominus muscle and inferiorly lacunar ligament. F: Flap; Eoa: External oblique aponeurosis.

oblique aponeurosis was closed by means of running $2 / 0$ polypropylene sutures. Finally skin was closed by simple interrupted $3 / 0$ polypropylene sutures.

\section{Results}

The average operating time was 50 minutes (ranged from 40 to 60 minutes). The follow-up period ranged from 8 to 12 months (mean, 10 months). Seroma was developed in the flap donor areas of one case. Seroma healed gradually with repeated aspiration. Except this case, others had not any complication as included wound infection, wound dehiscence, hematoma formation, paresthesia of the donor areas, stitch granuloma, chronic pain, testicular atrophy and hernia recurrence.

\section{Discussion}

The searches are going on for an ideal hernia repair but optimal treatment modality has not been achieved yet and recurrence has not been eliminated $[1,2,4]$. Various 
types of prosthetic materials which include polyester, mersilene, prolene, polypropylene, and expanded polytetrafluoroethylene meshes have been developed and commonly used for the reinforcement of the weakened abdominal wall $[2,3,5]$. Prosthetic materials allow the hernia to be repaired without tension being placed on the surrounding tissue. Excellent results have been achieved and reported recurrence rates have been less than 1 percent [4]. On the other hand these synthetic materials are physically and chemically inert, induce minimal inflammatory or foreign body reaction, resist mechanical strains, and cause seroma, testicular atrophy, chronic pain, and infection $[2,3,6,7]$. Avoiding for these complications, biomaterials which includes bioabsorbable polyglycolic acid and self reinforced polyglycolic acid have been developed and used in the experimental and clinical studies $[5,7]$. In addition to the all of these materials, dermal and fascial grafts have been used for abdominal wall reinforcement in experimental and clinical studies. The grafts may lead to deformity of the donor area [8-12]. Above all, abdominal wall layers have been used for its weakened areas, such as transversalis fascia and external oblique aponeurosis. The authors of these studies have reported minimal complications and $1.1 \%$ to $2 \%$ recurrence rate $[1,11]$. Furthermore, these techniques have not disadvantages of the prosthetic mesh.

We used innominate fascial flap for the inguinal hernias without mesh reinforcement. This flap is similar to the design of the aponeurofasciocutaneous groin flap, except not including external oblique aponeurosis and skin [13]. Innominate fascia (an external investing layer of the deep fascia), covers the superficial surface of the muscles and aponeurosis on the abdomen. Innominate fascia is supplied via deep branches of the superficial circumflex iliac artery [13]. This fascia is highly vascular and has an arborization of small vessels. Therefore, we used that fascia an island fascial flap for the repair of inguinal hernias our technique was performed via the open anterior approach.

Our study is similar to reconstruction of the inguinal hernias with abdominal wall layers such external oblique aponeurosis and transversalis fascia. Nevertheless we did not use component of the abdominal wall and our technique was less invasive. Therefore there is rather low possible of the complication. In addition, the flap can be harvested enough size and does not weak the abdominal wall.

\section{Conclusion}

In spite of having few cases and short time of follow-up, results of this work courage us. Because of our technique avoids from the complications and drawbacks of the prosthetic mesh, autograft and abdominal wall flaps, we think that the groin deep fascial flap can be a good alter- native to prosthetic meshes for reconstruction of inguinal hernia repair.

\section{REFERENCES}

[1] M. M. Moneer, “A New Preperitoneal Repair for Inguinal Hernia Using a Transpositined External Oblique Aponeurotic Flap," Surgery Today, Vol. 27, No. 11, 1997, pp. 1022-1025. doi:10.1007/BF02385782

[2] M. Kurzer, P. A. Belsham and A. E. Kark, "Prospective Study of Open Preperitoneal Mesh Repair for Recurrent Inguinal Hernia," British Journal of Surgery, Vol. 89, No. 1, 2002, pp. 90-93. doi:10.1046/j.0007-1323.2001.01956.x

[3] S. Petersen, G. Henke and L. Zimmermann, "Rectus Fascia Closure on Top of Mesh Hernia Repair in the Suplay Technique," Plastic and Reconstructive Surgery, Vol. 114, No. 7, 2004, pp. 1754-1760. doi:10.1097/01.PRS.0000142419.40722.C6

[4] P. Nordin, C. Bartelmess and C. Jansson, "Randomized Trial of Lichtenstein versus Sholdice Hernia Repair in General Surgical Practice," British Journal of Surgery, Vol. 89, No. 1, 2002, pp. 45-49. doi:10.1046/j.0007-1323.2001.01960.x

[5] N. W. Law, "A Comparison of Polypropylene Mesh, Expanded Polytetrafluoroethylene Patch and Polyglycolic Acid Mesh for the Repair of Experimental Abdominal Wall Defects," Acta Chirurgica Scandinavica, Vol. 156, No. 11-12, 1990, pp. 759-762.

[6] M. Korenkov, S. Sauerland and M. Arndt, "Randomized Clinical Trial of Suture Repair, Polyprolene Mesh or Autodermal Hernioplasty for Incisional Hernia," British Journal of Surgery, Vol. 89, No. 1, 2002, pp. 50-56. doi:10.1046/j.0007-1323.2001.01974.x

[7] A. Heino, A. Naukkarinen and T. Pietlainen, "Aplication of a Self-Reinforced Polyglycolic Acid (SR-RGA) Membrane to the Closure of an Abdominal Fascial Defect in Rats," Journal of Biomedical Materials Research, Vol. 48, No. 5, 1999, pp. 601-699.

doi:10.1002/(SICI)1097-4636(1999)48:5<596::AID-JBM 2>3.3.CO;2-J

[8] J. J. Disa, M. H. Klein and N. H. Goldberg, "Advantages of Autologous Fascia versus Synthetic Patch Abdominal Reconstruction in Experimental Animal Defects," Plastic and Reconstructive Surgery, Vol. 97, No. 4, 1996, pp. 801-806. doi:10.1097/00006534-199604000-00017

[9] J. J. Disa, M. H. Klein and N. H Goldberg, "Restoring Abdominal Wall Integrity in Contaminated Tissue-Deficient Wounds Using Autologous Fascia Grafts," Plastic and Reconstructive Surgery, Vol. 101, No. 4, 1998, pp. 979-986. doi:10.1097/00006534-199804040-00014

[10] C. Caloghera, A. Mihăilescu and P. Popovici, "De-Epidermized Free Skin Graft in the Treatment of Eventrations and Hernias," Revista de Chirurgie, Oncologie, Radiologie, o.r.l., Oftalmologie, Stomatologie. Chirurgie, Vol. 29, No. 2, 1980, pp. 93-100.

[11] M. M. Robert, B. Micheal and C. Mario, "Double Layer of Transversalis Fasca for Repair of Inguinal Hernia: Re- 
sults in 104 Cases," Surgery, Vol. 63, No. 3, 1968, pp. 423-429.

[12] V. N. Ianow, "Method of Dermal Autograft in 'Difficult Forms' of Inguinal Hernia," Vestnik Khirurgii Imeni I. I. Grekova, Vol. 160, No. 3, 2001, pp. 49-51.
[13] T. Ayyappan, A. Chandha and K. K. Porwal, "Axial Aponeurofasciocutaneous Groin Flap: An Extended Concept," Journal of Craniofacial Surgery, Vol. 12, No. 5, 2001, pp. 501-504. doi:10.1097/00001665-200109000-00020 